\title{
Reductions in Use of Colchicine after FDA Enforcement of Market Exclusivity in a Commercially Insured Population
}

\author{
Aaron S. Kesselheim, MD, JD, MPH ${ }^{1,3}$, Jessica M. Franklin, PhD ${ }^{1,3}$, Seoyoung C. Kim, MD, ScD, MSCE ",,3, \\ John D. Seeger, PharmD, DrPH ${ }^{1,3}$, and Daniel H. Solomon, MD, MPH
}

'Program On Regulation, Therapeutics, And Law (PORTAL), Division of Pharmacoepidemiology and Pharmacoeconomics, Department of Medicine, Brigham and Women's Hospital, Boston, MA, USA; ${ }^{2}$ Division of Rheumatology, Department of Medicine, Brigham and Women's Hospital, Boston, MA, USA; ${ }^{3}$ Harvard Medical School, Boston, MA, USA.

BACKGROUND: A brand-name version of colchicine (Colcrys) was introduced after its manufacturer conducted a clinical trial in acute gout patients, leading to higher prices for this drug.

OBJECTIVE: We analyzed the impact of the new singlesource colchicine product on prescribing and patient health spending as well as incidence rates of potentially dangerous concomitant use of clarithromycin and cyclosporine after formal FDA approval.

DESIGN/PARTICIPANTS: We conducted a retrospective cohort study of UnitedHealth-affiliated enrollees newly diagnosed with gout or FMF.

MAIN MEASURES: Among gout and FMF patients separately, we assessed linear trends in colchicine prescriptions, prescription drug costs, and total health care costs from 2009 to September 2010 (market exclusivity announced) compared to January 2011 (market exclusivity enforced) through 2012. Next, we estimated trends in coprescription within 15 days of clarithromycin, azithromycin (indicated on the Colcrys label for use in place of clarithromycin), and cyclosporine.

KEY RESULTS: Among gout patients, before Colcrys' market exclusivity, the odds of receiving colchicine within 30 days of gout diagnosis increased $1.4 \% /$ month (OR: 1.014, 95 \% CI: 1.011-1.018). Following FDA action, the odds decreased by $0.5 \% /$ month (OR: 0.995, 95 \% CI: 0.992-0.999) ( $p<0.001)$. Similarly, among FMF patients, odds of initiating colchicine changed from an increase of $2.8 \% /$ month to a decrease by $7.6 \% /$ month $(p=0.01)$. Patients receiving colchicine experienced increases in average monthly prescription drug costs (\$418 vs. \$651, $p<0.001)$ and health care costs $(\$ 3,406$ vs. $\$ 3,534$, $p<0.001)$. Incidence rates of colchicine/ clarithromycin co-prescription before and after FDA action did not change, while co-prescription of colchicine/cyclosporine increased after introduction of Colcrys [ -0.75 monthly change in patients $(95 \%$ CI: $-1.07,-0.43)$ vs. $0.13(95 \% \mathrm{CI}:-0.16,0.42)$, $p<0.001]$.

CONCLUSIONS: The FDA's actions were associated with a reduction in colchicine initiation and an increase in patient spending. By contrast, we did not observe any

Received September 5, 2014

Revised February 18, 2015

Accepted February 27, 2015

Published online April 9, 2015 association with improvements in avoidance of potentially dangerous co-prescriptions.

J Gen Intern Med 30(11):1633-8

DOI: $10.1007 / \mathrm{s} 11606-015-3285-7$

(C) Society of General Internal Medicine 2015

\section{INTRODUCTION}

In June 2006, the Food and Drug Administration (FDA) launched its unapproved drugs initiative to seek formal regulatory review of dozens of prescription drugs that had been on the market at the time the FDA first received authority to require new prescription drugs to be tested for efficacy and safety. ${ }^{1}$ The FDA's goal was to accomplish this process "without imposing undue burdens on consumers, or unnecessarily disrupting the market." 2 One of the drugs targeted by the FDA was colchicine, a therapeutic used to treat acute gout, as well as the rare inflammatory disease familial Mediterranean fever (FMF).

Following this initiative, a small controlled trial was conducted in 2007 confirming that colchicine was efficacious in managing acute gout. ${ }^{3}$ The trial randomized 184 patients to receiving placebo $(n=58)$, a low-dose colchicine regimen $(n=74)$ that had been recommended by professional society treatment guidelines, ${ }^{4}$ and a high-dose regimen $(n=52)$. After 1 week, gout patients in the low-dose regimen reported good symptom management and fewer adverse events than the longer regimen. The total effect size $(38 \%$ of patients receiving low-dose colchicine vs. $16 \%$ in the placebo arm) mirrored results from a previous trial of colchicine for acute gout $(73 \%$ vs. $36 \%)^{5}$ The trial concluded that the shortened dosing regimen (1.2 $\mathrm{mg}$ and $0.6 \mathrm{mg} 1 \mathrm{~h}$ later) was effective and safe. ${ }^{6}$

After reviewing the results from this trial, the FDA approved the brand-name colchicine (Colcrys) for use in US patients with acute gout and granted the manufacturer a guaranteed period of 3 years of market exclusivity. ${ }^{7}$ The manufacturer also received 7 years of exclusivity under the Orphan Drug Act for the drug's use in FMF, although no new studies were conducted in FMF patients. ${ }^{8}$ Colcrys was sold for approximately $\$ 5$ per tablet, a substantial increase over the average of $\$ 0.09$ per tablet for which the unapproved version of prescription colchicine was previously sold by numerous 
manufacturers. ${ }^{9}$ In a subsequent letter, the FDA justified its actions by pointing to safety concerns with these unapproved colchicine formulations, in particular deaths associated with concomitant use of colchicine with the antibiotic clarithromycin, noting that this approved version of colchicine would be required to include information in its drug label warning physicians about the potential danger of colchicine/ clarithromycin interactions. ${ }^{10,11}$ In fact, the label suggested to physicians that azithromycin should be prescribed in place of clarithromycin in patients requiring Colcrys. ${ }^{12}$

Despite the FDA's initial approval of Colcrys on 30 July 2009, some manufacturers making unapproved colchicine continued to produce the drug until the FDA issued an order in September 2010 giving them 90 days to stop manufacturing their products. ${ }^{13}$ Thus, in January 2011, Colcrys became the only colchicine formulation in the US. ${ }^{14}$ The impact of this FDA order on trends in colchicine prescribing and drug costs remains unknown.

\section{METHODS}

We evaluated the effect of the FDA-mandated change from multisource to single-manufacturer production of colchicine in the US on prescriptions of the drug for patients with gout or FMF diagnoses, and on rates of co-prescriptions of colchicine and clarithromycin and cyclosporine, two drugs for which specific co-prescription warnings were provided in the new Colcrys label. Our data source was the Optum Research Database, which contains medical and pharmacy data on insurance claims for more than 13 million current beneficiaries affiliated with the UnitedHealth commercially insured population. ${ }^{15}$ This source population reflects the nationwide geographic distribution of the health insurer and has demographics similar to the US census age distribution for gender and age groups $<65$ years. Between 2011 and 2012, UnitedHealth removed colchicine from Tier 1 (lowest cost, for generic products) on its formulary and added Colcrys to Tier 2 (midrange cost, for preferred brand-name products). ${ }^{16,17}$

We identified enrollees in the Optum database who were newly diagnosed with gout (diagnosis codes $274 . \mathrm{XX} / \mathrm{V} 77.5$ or uric acid level $>6.0 \mathrm{mg} / \mathrm{dl})$ or FMF (277.31/713.7) from January 2009-December 2012 without the patient having any such diagnoses or uric acid level in the prior 365 days of health plan enrollment. Users included those who filled a prescription for any version of colchicine within the 30 days after their index date. We tabulated the percentages of colchicine users and non-users among newly diagnosed patients in each month of the study period, as well as the total number of colchicine users and clarithromycin users in the Optum database, and the average monthly prescription drug costs generated by patients who were new users of colchicine.

First, we estimated a discontinuous logistic regression model separately in patients newly diagnosed with gout and FMF. The dependent variable was a binary indicator for receiving colchicine within 30 days after diagnosis, and independent variables were constructed based on the month of diagnosis in order to estimate the linear trend in the log odds of receiving colchicine, separately from January 2009 to September 2010 and from January 2011 to December 2012, as shown in the model, $\operatorname{Logit}\left\{\operatorname{Pr}\left(\mathrm{Y}_{\mathrm{i}}=1\right)\right\}=\mathrm{b}_{0}+$ $b_{1} T_{1 i}+b_{2} T_{2 i}+b_{3}$ Post $_{i}$, where $Y$ is the indicator of colchicine dispensing, $T_{1}$ is a time variable that represents when each patient was diagnosed with gout as the number of months after January $2009, \mathrm{~T}_{2}$ is a time spline that begins in January 2011, and Post is a binary indicator of being diagnosed in January 2011 or after. The 90-day skip period corresponds to the transition time provided in the FDA's order enforcing Colcrys' market exclusivity. ${ }^{13}$ This model was reestimated adjusting for patient characteristics including age, gender, Charlson score, the number of inpatient visits, and the number of physician visits, calculated during the year prior to diagnosis. T-tests were used to compare average prescription drug costs before and after the FDA action among both gout and FMF patients who initiated colchicine.

Finally, we used linear regression to estimate the change over time in total patients per month receiving colchicine prescriptions who also filled clarithromycin within 15 days, adjusting for background prevalent use of colchicine and clarithromycin each month (overall number of users per month), as shown in the model, $\mathrm{Y}_{\mathrm{t}}=\mathrm{b}_{0}+\mathrm{b}_{1} \mathrm{~T}_{1 \mathrm{t}}+\mathrm{b}_{2} \mathrm{~T}_{2 \mathrm{t}}+\mathrm{b}_{3}$ Post $_{\mathrm{t}}+\mathrm{b}_{4}$ Colch $_{\mathrm{t}}+\mathrm{b}_{5}$ Clar $_{\mathrm{t}}$, where $\mathrm{Y}$ is the number of patients receiving co-prescription in each month, $\mathrm{T} 1$ is a time variable that represents the number of months after January 2009, T2 is a time spline that begins in January 2011, Post is a binary indicator of the period after the FDA order, Colch is the number of patients receiving any prescription for colchicine in each month, and Clar is the number of patients receiving any prescription for clarithromycin. This adjustment allowed the trends in co-prescription to be isolated from the overall trends in the use of each drug. We did the same for co-prescriptions of colchicine with azithromycin, which may be expected to rise in the context of a clarithromycin-related warning, because the Colcrys drug label identified azithromycin as a safe potential substitute for clarithromycin with minimal drug-drug interactions. ${ }^{18}$ We also examined co-prescriptions of colchicine with cyclosporine, which may be expected to fall because of a warning similar to the colchicine/clarithromycin one.

The correlation of repeated measures of the percentage of patients receiving colchicine over time was effectively zero; therefore, analyses that adjusted for this correlation were identical to the results in our main analysis.

\section{RESULTS}

The full cohort included 216,640 individuals, of which 630 $(0.2 \%)$ met entry criteria by virtue of an FMF diagnosis. The cohort was $76 \%$ male, had a mean age of 52 (SD 12.9), and averaged 0.15 inpatient (SD 0.56) and 8.0 physician visits (SD $11.3)$ in the year before the index date. The pre- and post-FDA order cohorts had similar demographic characteristics.

Focusing on patients newly diagnosed with gout, before the FDA order, the odds of receiving colchicine within 30 days of 
diagnosis increased $1.4 \%$ month [odds ratio (OR): 1.014, $95 \%$ confidence interval (CI): 1.011-1.018]. Following the FDA action, the odds decreased by $0.5 \%$ month (OR: $0.995,95 \%$ CI: 0.992-0.999, $p<0.001$ for difference in trends). Between September 2010 and January 2011, the odds of initiating colchicine within 30 days of diagnosis dropped by $16 \%$ (OR: 0.84 , $95 \%$ CI: 0.80-0.89) (Fig. 1a). After adjusting for patient characteristics, trends were nearly identical before the FDA order (OR: 1.015, $95 \%$ CI: 1.012-1.019), after the order (OR: 0.996, 95 \% CI: 0.993-1.000), and between September 2010 and January 2011 (OR: 0.84, 95 \% CI: 0.80-0.89).

Among patients newly diagnosed with FMF, the odds of receiving colchicine within 30 days of diagnosis increased $2.8 \%$ /month [odds ratio (OR): 1.028, $95 \%$ confidence interval (CI): 0.971-1.087]. Following the FDA action, the odds decreased by $7.6 \%$ /month (OR: 0.926, $95 \%$ CI: 0.866-0.978, $p=0.01$ for difference in trends) (Fig. 1b).

Patients' total prescription drug costs increased after the FDA action. From January 2009 to September 2010, the average monthly total prescription drug costs were $\$ 418$ as compared to $\$ 651$ between January 2011 and December 2012 $(p<0.001)$.

Figure 2 depicts the proportion of all colchicine patients each month who received a co-prescription of clarithromycin during our study time period. In contrast to the trends in prescribing and drug costs, the trend in concurrent prescriptions of colchicine and clarithromycin was falling both before and after the FDA action, with no significant change in the rate of decline [ -0.06 adjusted average monthly change in number of patients (95\% CI: -0.35 , 0.22 ) vs. -0.10 (95\% CI: $-0.36,0.15), p=0.78$ for difference in
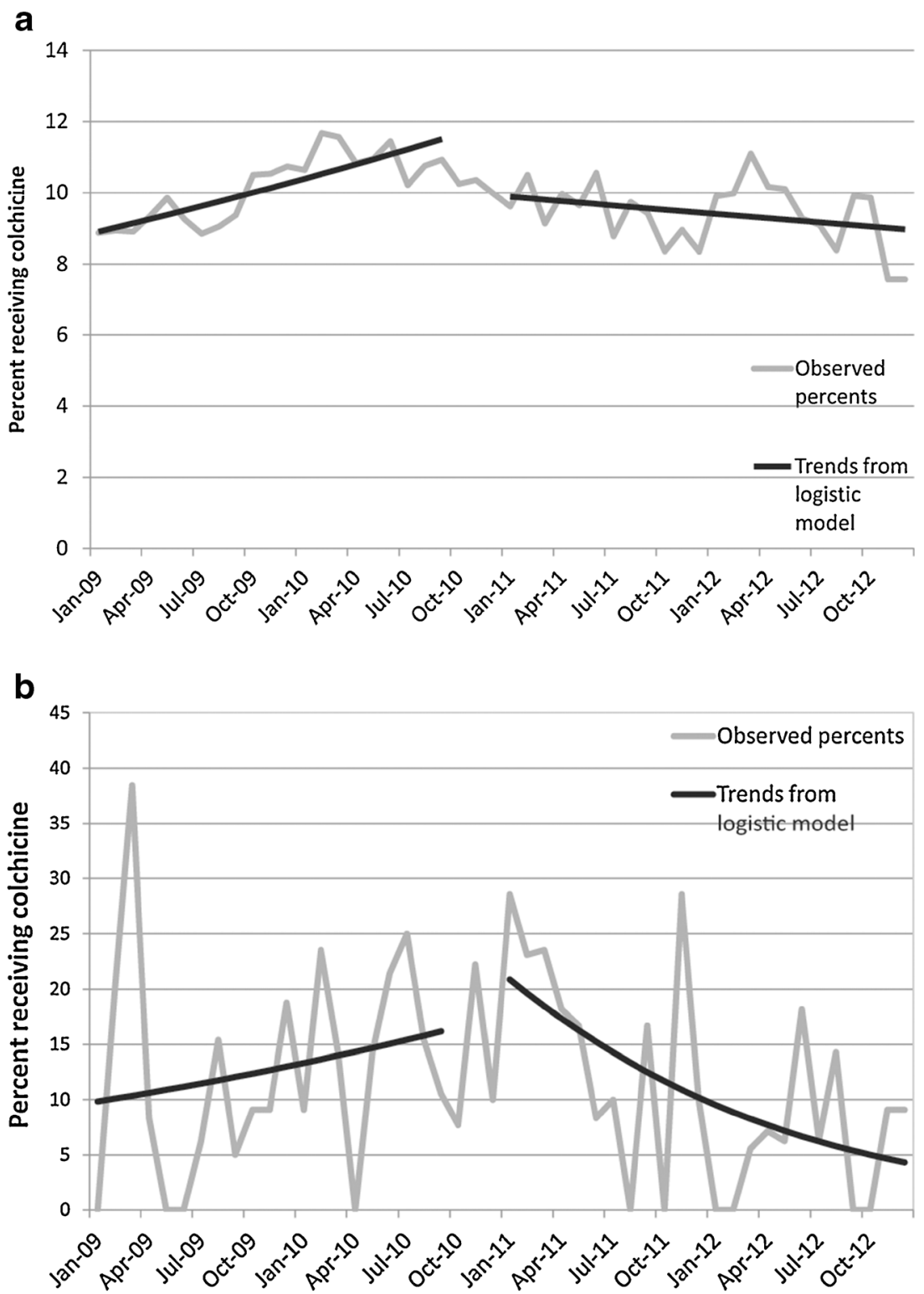

Figure 1 a Percent of newly diagnosed gout patients receiving colchicine, 2009-2012. b Percent of newly diagnosed FMF patients receiving colchicine, 2009-2012. Legend: This figure shows the raw percent of newly diagnosed gout (a) and FMF (b) patients initiating colchicine each month from January 2009-December 2012, along with the trends in initiation, as estimated from the discontinuity logistic regression model 


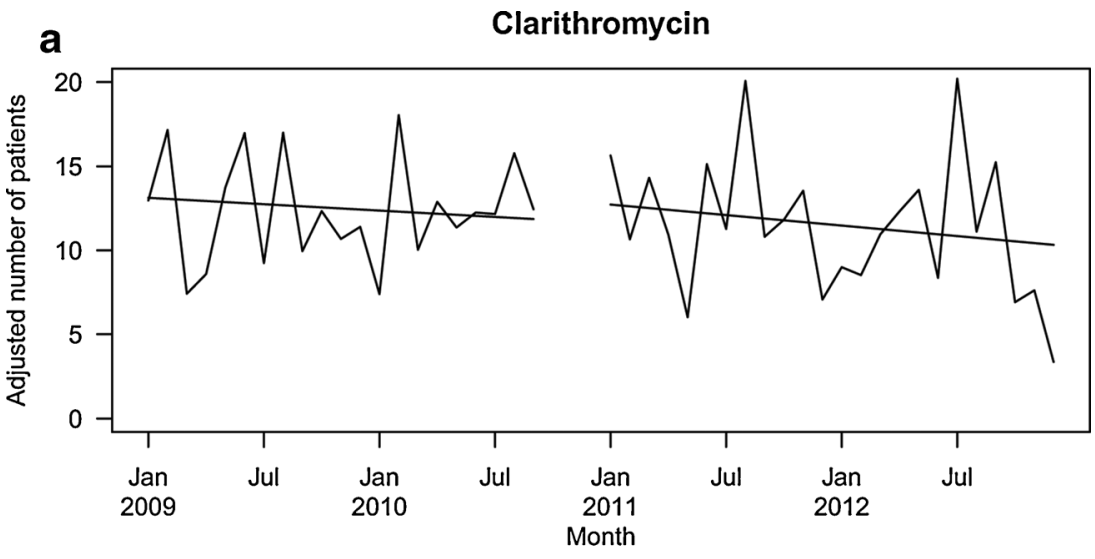

b

Azithromycin

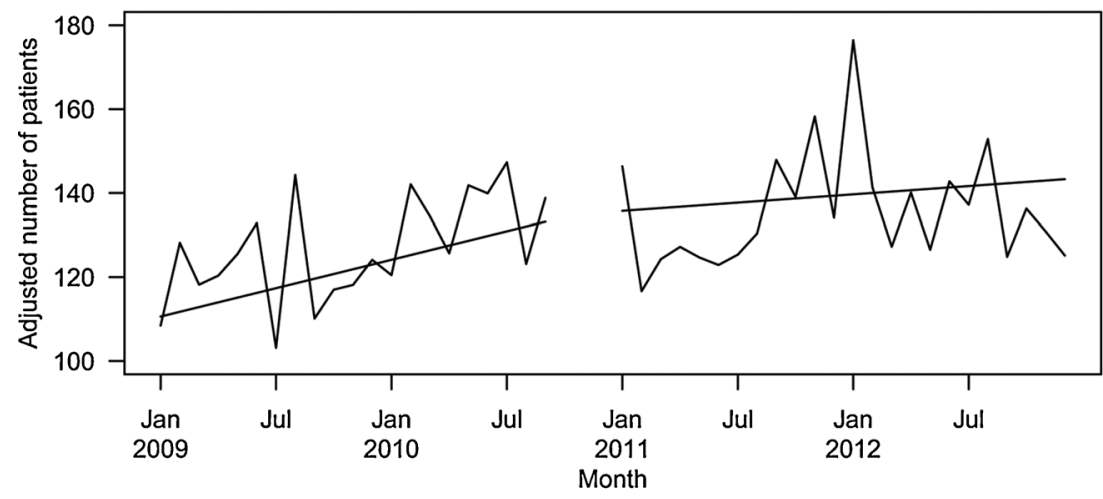

C

Cyclosporine

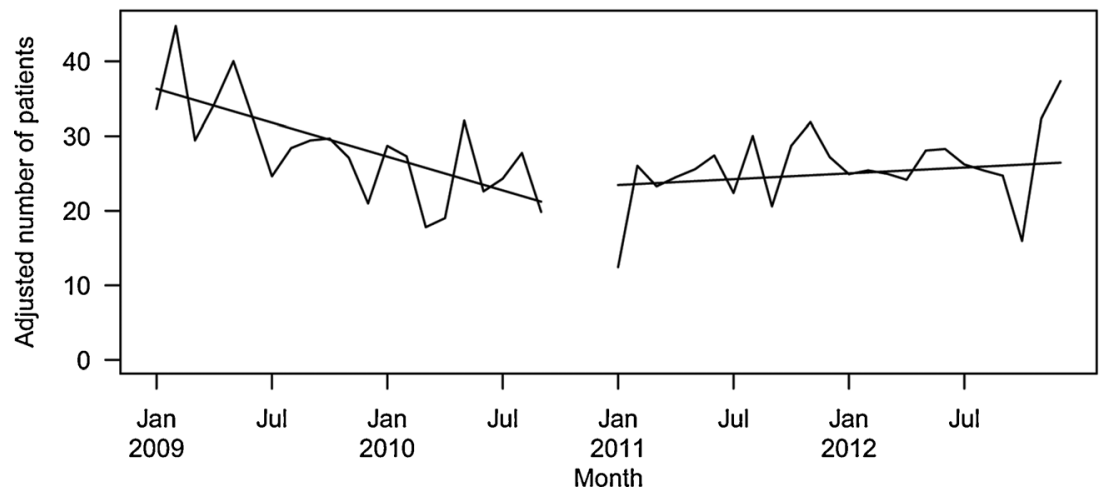

Figure 2 Panel a Number of patients receiving concomitant prescriptions of colchicine and clarithromycin, adjusted for the overall use of each drug over time, 2009-2012. Panel b Analysis for concomitant prescriptions of colchicine and azithromycin, 2009-2012. c Analysis for concomitant prescriptions of colchicine and cyclosporine, 2009-2012. Legend: This figure shows the number of patients who received a concomitant prescription for clarithromycin (a), azithromycin (b), and cyclosporine (c) within 15 days of a colchicine prescription, adjusted for the background trends in use for each drug, along with the adjusted trends in concomitant use, as estimated from the discontinuity model. In the raw data, adjustment was carried out by standardizing the number of concomitant prescriptions observed each month based on the average number of prescriptions per month for each drug over the entire study period

trends] (Fig. 2a). Our additional co-prescribing analyses show no significant difference in the rates of co-prescription of colchicine with azithromycin [1.1 adjusted average monthly change in number of patients $(95 \% \mathrm{CI}: 0.09,2.17)$ vs. $0.33(95 \% \mathrm{CI}$ : $-0.56,1.21], p=0.25$ for difference in trends] before and after the skip period relating to the time of FDA action and a significant increase in co-prescription of colchicine with cyclosporine $[-0.75$ adjusted average monthly change in number of patients $(95 \% \mathrm{CI}$ :
$-1.07,-0.43$ ) vs. 0.13 (95 \% CI: $-0.16,0.42), p<0.001$ for difference in trends] (Fig. 2b-c).

\section{DISCUSSION}

We sought to evaluate the impact on a large commercially insured population of the FDA's actions related to colchicine, a 
drug that had been widely used for gout and sold at low cost for many years in the US because it predated the FDA's regulatory authority over prescription drug efficacy and safety. ${ }^{19}$ After a small trial showed safe and efficacious use of colchicine in a lowintensity dosing regimen among patients with acute gout, the FDA granted market exclusivity for the manufacturer, leading to a substantial price increase. In response, UnitedHealth - as well as other major insurers such as Aetna ${ }^{20}$ and Cigna $^{21}$ - increased the co-payments for patients receiving a prescription of the drug. The outcome, documented in this study, was a reduction in initiations of colchicine and an increase in prescription drug costs generated by patients starting Colcrys. Our study was limited to a single insured population with co-payment arrangements as part of its prescription drug benefits and thus may underestimate the impact of the FDA's actions in populations with less favorable drug cost-sharing schemes.

Although we did not assess patient outcomes, the public health implications of these findings are potentially substantial. Diagnoses of gout - the primary indication for which colchicine is used - have been increasing in the US in recent years. ${ }^{22}$ Frequent flares of gout and tophi resulting from persistently elevated uric acid levels are associated with adverse physical and social consequences. When Colcrys reached the market, numerous patients, ${ }^{23}$ providers,${ }^{24}$ and professional organizations ${ }^{25}$ worried how its higher price would affect health care delivery, ${ }^{26}$ because colchicine has long been known as an effective therapy for acute attacks of gouty arthritis. Reduction in its use may contribute to excessive reliance on alternative therapies such as non-steroidal anti-inflammatory drugs or corticosteroids - which both have important risks of their $\mathrm{own}^{27}$-and unnecessary office or hospital visits by patients. In addition, anecdotal reports suggested that some patients who switched from previously available unapproved brands to the FDA-approved version experienced recurrence of adverse events because of the change in formulation. ${ }^{28}$ Further research is necessary to identify whether there was a change in rates of colchicine-related adverse events, such as gastrointestinal toxicity, among more limited numbers of patients initiating colchicine during this time period.

It is unlikely that the FDA approval of colchicine inspired more physicians to use it in concert with cyclosporine. A more likely explanation is that during the time period of our study, the annual number of transplantations was increasing, particularly for kidney transplants, ${ }^{29}$ which are known to be associated with hyperuricemia and gout. ${ }^{30}$ Transplant physicians may decide with their patients that the benefits of co-prescription outweigh the risks. Alternatively, the FDA or other public health authorities may need to better publicize the concerning interaction with cyclosporine that is formally in colchicine's label.

The public health implications of our findings are potentially more acute among patients with FMF for whom colchicine is the primary therapeutic option. Reports have suggested that hardships among this rare disease population are due to the introduction of Colcrys and a concomitant increase in price. ${ }^{24}$ The manufacturer offers a patient assistance program to help patients with the costs of Colcrys, but these programs often have complicated qualifying criteria and do not represent a dependable safety net. ${ }^{31}$

A leading justification for these changes was the enhanced safety that a single, FDA-approved version of colchicine would provide. However, we saw no change in the incidence of coprescriptions of colchicine and clarithromycin, the key interaction of interest to the FDA. While we do not know whether patients actually took the medications they were prescribed, it is interesting that co-prescription of these two medications appears to have been falling throughout the study period. The reason for the drop in concomitant use is unclear but may be related to greater uptake during these years of electronic medical record systems and other tools by both physicians and pharmacists that provide automated safety alerts related to specific drug-drug interactions, such as colchicine-clarithromycin, an interaction that had been welldocumented by that time. ${ }^{32}$ This explanation accounts for the steady rise in colchicine-azithromycin co-prescriptions during the same time period, with no effect from the switch of the market to Colcrys. However, further research into the reason why colchicine and cyclosporine co-prescriptions appear to be rising in recent years in direct contradiction to the labeled warning about interactions between the two drugs is needed.

Our analysis is limited by the possibility that other policy changes affecting colchicine use may have been occurring during the same time period that were unknown to us. For example, we cannot rule out other contributors to the observed trends, such as new professional society guidelines for gout management disseminated by the American College of Rheumatology during our study period. ${ }^{33}$ However, time trend analyses control for population characteristics that affect drug utilization as long as the population distribution does not change significantly over time, which is often a reasonable assumption in a large, insured population such as the one we have used. To further explore the potential for changes in population characteristics over time, we repeated models adjusting for patient demographics and medical characteristics and found no impact on estimated time trends

Data from the transition from colchicine to Colcrys may be instructive to the FDA as it continues its unapproved drugs initiative. The FDA's goals in attempting to strengthen its regulatory oversight related to unapproved drugs were laudable, but our study found that at least one expected public health benefit - changes in dangerous co-prescriptions - did not occur in the case of colchicine. By contrast, it is clear that the FDA action resulted in a reduction in colchicine initiation and an increase in cost.

Conflict of Interest: Dr. Kesselheim is supported by a Greenwall Faculty Scholarship in Bioethics. Dr. Kim is supported by the NIH grant K23 AR059677. She received a research grant from Pfizer and tuition support for the Pharmacoepidemiology Program at the Harvard School of Public Health partially funded by the Pharmaceutical Research and Manufacturers of America (PhRMA). Dr. Solomon is funded by a Mentoring Award from the NIH (K24 AR055989). The authors have no conflicts of interests to report. 
Corresponding Author: Aaron S. Kesselheim, MD, JD, MPH; , 1620 Tremont St., Suite 3030, Boston, MA 02120, USA (e-mail: akesselheim@partners.org).

\section{REFERENCES}

1. Carpenter D. Reputation and Power: Organizational Image and Pharmaceutical Regulation at the FDA. Princeton: Princeton Univ Press; 2010.

2. Food and Drug Administration. Unapproved drugs initiative. Jan 52011 http://www.fda.gov/Drugs/GuidanceComplianceRegulatoryInformation/ EnforcementActivitiesbyFDA/SelectedEnforcementActionson UnapprovedDrugs/ucm118990.htm.

3. Terkeltaub RA, Furst DE, Bennett K, Kook KA, Crockett RS, Davis MW. High versus low dosing of oral colchicine for early acute gout flare: twentyfour-hour outcome of the first multicenter, randomized, double-blind, placebo- controlled, parallel-group, dose-comparison colchicine study. Arthritis Rheum. 2010;62(4): 1060-8.

4. Zhang W, Doherty M, Bardin T, Pascual E, Barskova V, Conaghan P, Gerster J, Jacobs J, Leeb B, Liote F, McCarthy G, Netter P, Nuki G, Perez-Ruiz F, Pignone A, Pimentao J, Punzi L, Roddy E, Uhlig T, Zimmermann-Gorska I. EULAR evidence based recommendations for gout, part II: management. Ann Rheum Dis. 2006;65(10):1312-24.

5. Ahern MJ, Reid C, Gordon TP, McCredie M, Brooks PM, Jones M. Does colchicine work? The results of the first controlled study in acute gout. Aust NZ J Med. 1987;17(3):301-4.

6. Kesselheim AS, Solomon DH. Author Reply re: Incentives for drug development-the curious case of colchicine. N Engl J Med. 2010;363(15): 1484-5.

7. Rockoff JD. An old gout drug gets new life and a new price, riling patients. Wall St. J. 12 Apr 2010.

8. Center for Drug Evaluation and Research. Application number 22-352: medical review. 24 Nov 2008. Available at: http://www.accessdata.fda.gov/ drugsatfda_docs/nda/2009/022352s000_MedR.pdf.

9. Kesselheim AS, Solomon DH. Incentives for drug development-the curious case of colchicine. N Engl J Med. 2010;362(22):2045-7.

10. Woodcock J, Okada $\mathbf{S}$. Letter to the Editor re: Incentives for drug development-the curious case of colchicine. N Engl J Med. 2010;363(15): 1484 .

11. Terkeltaub RA, Furst DE, Digiacinto JL, Kook KA, Davis MW. Novel evidence-based colchicine dose-reduction algorithm to predict and prevent colchicine toxicity in the presence of cytochrome P450 3A4/P-glycoprotein inhibitors. Arthritis Rheum. 2011;63(8):2226-37.

12. AR Scientific Inc. Highlights of prescribing information: colchicine (Colcrys). Jul 2009. Available at: http://www.accessdata.fda.gov/ drugsatfda_docs/label/2009/022352lbl.pdf.

13. Food and Drug Administration. FDA orders halt to marketing of unapproved single-ingredient oral colchicine. 30 Sept 2010. Available at: http://www.fda.gov/NewsEvents/Newsroom/PressAnnouncements/ ucm227796.htm.

14. Guglielmo BJ. The colchicine debacle. JAMA Intern Med. 2013;173(3):184-5

15. Eng PM, Johannes CB, Chiang CC, West WA, Seeger JD, Walker AM. Survey of disability, medication use, and medication satisfaction among members with rheumatoid arthritis of a large health care plan database. Pharmacoepidemiol Drug Saf. 2005;14:S1-218.
16. UnitedHealthcare. Your 2012 prescription drug list: quick-reference guide. Sept 2011.

17. UnitedHealthcare. 2011 three-tier prescription drug list: consumer reference guide. Sept 2010.

18. Westphal JF. Macrolide-induced clinically relevant drug interactions with cytochrome P-450A (CYP) 3A4: an update focused on clarithromycin, azithromycin and dirithromycin. $\mathrm{Br} \mathrm{J}$ Clin Pharmacol. 2000;50(4):285-95.

19. Food and Drug Administration. FDA approves colchicine for acute gout, Mediterranean fever. 30 Jul 2009. Available at: www.fda.gov/newsevents/ newsroom/pressannouncements/ucm174620.htm

20. Aetna. 2013 Aetna comprehensive formulary drug list (base). September 2013. Available at: http://www.aetnamedicare.com/documents/individual/2013/formularies/2013_medicare_cvs_essentials_preferred_drug_list. pdf.

21. Cigna Healthcare. Drug list search results. Available at: https://my.cigna. com/teamsite/cgi-bin/customer_care/member/drug_list/DrugList.cgi? search_by=name\&LeanIndicator=\&referer=\&Pid=\#Cdrugs.

22. Zhu Y, Pandya BJ, Choi HK. Prevalence of gout and hyperuricemia in the US general population: the National Health and Nutrition Examination Survey 2007-2008. Arthritis Rheum. 2011;63(10):313641.

23. Meyer H. Is FDA approval for safety...or price gouging? LA Times. 18 Jan 2010.

24. Grody Ww, Getzug T. Colchicine's other indication-effect of FDA action. N Engl J Med. 2010;363(23):2267-8.

25. Uliman K. Colcrys approval triggers questions. The Rheumatologist. May 2010. Available at: http://www.the-rheumatologist.org/details/article/ 865591/Colcrys_Approval_Triggers_Questions.html.

26. Rosenthal E. The soaring cost of a single breath. NY Times. 12 Oct 2013.

27. Janssens HJ, Lucassen PL, Van de Laar FA, Janssen M, Van de Lisdonk EH. Systematic corticosteroids for acute gout. Cochrane Database Syst Rev. 2008;2, CD005521.

28. Jagger J. Survey report: comparative colchicine brand response in FMF patients. Eurordis. 10 Apr 2011. Available at: http://download.eurordis. org/documents/pdf/FMFSurveyallcases_latest.pdf.

29. National Institute of Diabetes and Digestive and Kidney Diseases. Kidney disease statistics for the United States. 15 Nov 2012. Available at: http://kidney.niddk.nih.gov/kudiseases/pubs/ kustats/\#11.

30. Stamp LK, Chapman PT. Gout and organ transplantation. Curr Rheumatol Rep. 2012;14(2): 165-72.

31. Howard DH. Drug companies' patient-assistance programs-helping patients or profits? N Engl J Med. 2014;371(2):97-9.

32. Hung IF, Wu AK, Cheng VC, Tang BS, To KW, Yeung CK, Woo PC, Lau SK, Cheung BM, Yuen KY. Fatal interaction between clarithromycin and colchicine in patients with renal insufficiency: a retrospective study. Clin Infect Dis. 2005;41(3):291-300.

33. Khanna D, Fitzgeralf JD, Khanna PP, Bae S, Signh MK, Neogi T, Pillinger MH, Merill J, Lee S, Prakash S, Kaldas M, Gogia M, Perez-Ruiz F, Taylor W, Liote F, Choi H, Singh JA, Dalbeth N, Kaplan S, Niyyar V, Jones D, Yarows SA, Roessler B, Kerr G, King C, Levy G, Furst DE, Edwards NL, Mandell B, Schumacher HR, Robbins M, Wenger N, Terkeltaub R. 2012 American College of Rheumatology guidelines for management of gout. Part 1: systematic nonpharmacologic and pharmacologic therapeutic approaches to hyperuricemia. Arthritis Care Res. 2012;64(10): 1431-46. 